\title{
Generalized Kronecker Product and Its Application
}

\author{
Xingxiang Liu \\ School of mathematics and computer Science \\ Yanan University \\ Shanxi 716000, China \\ E-mail: 1xx6407@163.com
}

\begin{abstract}
In this paper, we promote the definition of Kronecker product, and give its corresponding properties. As the application of generalized Kronecker product, this paper shows the determination method that the algebraic operation in finite set suits the associative law.
\end{abstract}

Keywords: Generalized Kronecker product, Assoziativitaet, Decision condition

\section{Introduction}

Kronecker product expresses a special product of matrix. The product of a matrix $A$ by $m \times n$ and a matrix $B$ by $p \times q$ can be denoted by $A \otimes B$, which is a matrix by $m p \times n q$.

Definition 1.(Kronecker product) ${ }^{(B e l l m a n R ., 1970)}$ The product of a matrix $A$ by $m \times n$ and a matrix $B$ by $p \times q$ can be denoted by $A \otimes B$, which is defined as follows:

$$
A \otimes B=\left[a_{i, j} B\right]=\left[\begin{array}{cccc}
a_{11} B & a_{12} B & \cdots & a_{1 n} B \\
a_{21} B & a_{22} B & \cdots & a_{2 n} B \\
\vdots & \vdots & \ddots & \vdots \\
a_{m 1} B & a_{m 2} B & \cdots & a_{m n} B
\end{array}\right] .
$$

Kronecker product also can be called direct product or tensor product.

Kronecker product has the following properties:

1). For $A_{m \times n}$ and $B_{p \times q}$, generally $A \otimes B \neq B \otimes A$.

2). The Kronecker product of arbitrary matrix and zero matrix equals zero matrix, i.e. $A \otimes 0=0 \otimes A=0$.

3). If $\alpha$ and $\beta$ are constant, $\alpha A \otimes \beta B=\alpha \beta(A \otimes B)$.

4). For $A_{m \times n}, B_{n \times k}, C_{l \times p}$ and $D_{p \times q}, A B \otimes C D=(A \otimes C)(B \otimes D)$.

5). For $A_{m \times n}, B_{p \times q}, C_{p \times q}, A \otimes(B \pm C)=(A \otimes B) \pm(A \otimes C),(B \pm C) \otimes A=(B \otimes A) \pm(C \otimes A)$.

Note: properties 1)-5) is referred from (Rao c R,1971).

6). For $A_{m \times n}$ and $B_{p \times q},(A \otimes B)^{T}=A^{T} \otimes B^{T}$.

7). For $A_{m \times n}$ and $B_{p \times q}, \operatorname{rank}(A \otimes B)=\operatorname{rank}(A) \operatorname{rank}(B)$.

8). For $A_{m \times m}$ and $B_{n \times n}, \operatorname{det}(A \otimes B)=(\operatorname{det} A)^{n}(\operatorname{det} B)^{n}$.

9). For $A_{m \times m}$ and $B_{n \times n}, \operatorname{tr}(A \otimes B)=\operatorname{tr}(A) \operatorname{tr}(B)$.

10). For $A_{m \times n}, B_{m \times n}, C_{p \times q}$ and $D_{p \times q},(A+B) \otimes(C+D)=A \otimes C+A \otimes D+B \otimes C+B \otimes D$. 
11). For $A_{m \times n}, B_{k \times l}, C_{p \times q}$ and $D_{r \times s},(A \otimes B) \otimes(C \otimes D)=A \otimes B \otimes C \otimes D$.

12). For $A_{m \times n}, B_{k \times l}, C_{p \times q},(A \otimes B) \otimes C=A \otimes(B \otimes C)$.

13). For $A_{m \times n}, B_{p \times q}, C_{n \times r}$ and $D_{q \times s},(A \otimes B)(C \otimes D)=(A \otimes C)(B \otimes D)$.

Note: properties 6)-13) is referred from (Brewer j w, 1978, 772-781).

But for the need of the real life and the mathematics development, the element of matrix may not limit to numbers, thus we introduce a new concept, also namely promoting the matrix concept.

\section{Definition of generalized Kronecker product}

Definition 2. Suppose $S$ be a nonempty set, $(S, \circ,+)$ be an algebra system, then $A$ is matrix in algebraic system $(S, \circ,+)$ if and only if $A=\left[a_{i j}\right]_{m \times n}, a_{i j} \in S(i=1,2, \cdots, m ; j=1,2, \cdots, n)$.

Definition of Kronecker product can be promoted as the below definition.

Definition 3(generalized Kronecker product). The Kronecker product of a matrix $A$ by $m \times n$ and a matrix $B$ by $p \times q$ in algebra system $(S, \circ,+)$ can be denoted by $A \otimes B$, which is defined as follows:

$$
A \otimes B=\left[a_{i j} \circ B\right]=\left[\begin{array}{cccc}
a_{11} \circ B & a_{12} \circ B & \cdots & a_{1 n} \circ B \\
a_{21} \circ B & a_{22} \circ B & \cdots & a_{2 n} \circ B \\
\vdots & \vdots & \ddots & \vdots \\
a_{m 1} \circ B & a_{m 2} \circ B & \cdots & a_{m n} \circ B
\end{array}\right] .
$$

Note: The generalized product, addition and number product of matrix are similar with the usual product, addition and number product of matrix.

\section{Properties of generalized Kronecker product}

Theorem 1. For $A_{m \times n}$ and $B_{p \times q}$, generally $A \otimes B \neq B \otimes A$.

Since the number operate is a special algebraic operator, for restricted untenable proposition, it is also untenable in generalized condition.

Theorem 2. The Kronecker product of arbitrary matrix and zero matrix equals zero matrix, i.e. $A \otimes 0=0 \otimes A=0$.

The reason is as that of theorem 1.

Theorem 3. If $(S, \circ)$ is a commutative semi-group, and for arbitrary $\alpha$ and $\beta,(\alpha \circ A) \otimes(\beta \circ B)=(\alpha \circ \beta) \circ(A \otimes B)$.

This theorem is equivalent the operate of elements: $\left(\alpha \circ a_{i, j}\right) \circ\left(\beta \circ b_{k l}\right)=(\alpha \circ \beta) \circ\left(a_{i j} \circ b_{k l}\right)$, where $a_{i j}=$ $[A]_{i j}, b_{k l}=[B]_{k l}$.

Theorem 4. If $(S, \circ)$ is a commutative semi-group, and for $A_{m \times n}, B_{n \times k}, C_{l \times p}$ and $D_{p \times q},(A \circ B) \otimes(C \circ D)=$ $(A \otimes C) \circ(B \otimes D)$.

This theorem is equivalent the operate of elements: $\left(a_{i j} \circ b_{k l}\right) \circ\left(c_{t u} \circ d_{w v}\right)=\left(a_{i, j} \circ c_{t u}\right) \circ\left(b_{k l} \circ d_{w v}\right)$, where $a_{i j}=[A]_{i j}, b_{k l}=[B]_{k l}, c_{t u}=[C]_{t u}, d_{w v}=[D]_{w v}$.

Theorem 5. If $(S,+, \circ)$ is a ring, and for $A_{m \times n}, B_{p \times q}, C_{p \times q}, A \otimes(B \pm C)=(A \otimes B) \pm(A \otimes C),(B \pm C) \otimes A=$ $(B \otimes A) \pm(C \otimes A)$.

Theorem 6. If $(S, \circ)$ is a commutative algebraic system, and for $A_{m \times n}$ and $B_{p \times q},(A \otimes B)^{T}=A^{T} \otimes B^{T}$.

Theorem 7. If $(S,+, \circ)$ is a ring and for $A_{m \times n}, B_{m \times n}, C_{p \times q}$ and $D_{p \times q},(A+B) \otimes(C+D)=A \otimes C+A \otimes D+B \otimes C+B \otimes D$.

This theorem is equivalent the operate of elements: $\left(a_{i j}+b_{i j}\right) \circ\left(c_{k l}+d_{k l}\right)=a_{i j} \circ c_{k l}+a_{i j} \circ d_{k l}+b_{i j} \circ c_{k l}+b_{i j} \circ d_{k l}$, where $a_{i j}=[A]_{i j}, b_{i j}=[B]_{i j}, c_{k l}=[C]_{k l}, d_{k l}=[D]_{k l}$.

Theorem 8. If $(S, \circ)$ is a commutative semi-group, and for $A_{m \times n}, B_{k \times l}, C_{p \times q}$ and $D_{r \times s},(A \otimes B) \otimes(C \otimes D)=$ $A \otimes B \otimes C \otimes D$.

This theorem is equivalent the operate of elements: $\left(a_{i j} \circ b_{k l}\right) \circ\left(c_{t u} \circ d_{w v}\right)=a_{i j} \circ b_{k l} \circ c_{t u} \circ d_{w v}$, where $a_{i j}=[A]_{i j}, b_{k l}=[B]_{k l}, c_{t u}=[C]_{t u}, d_{w v}=[D]_{w v}$.

Theorem 9. If $(S, \circ)$ is a semi-group, and for $A_{m \times n}, B_{k \times l}, C_{p \times q},(A \otimes B) \otimes C=A \otimes(B \otimes C)$. 
This theorem is equivalent the operate of elements: $\left(a_{i j} \circ b_{k l}\right) \circ c_{t u}=a_{i j} \circ\left(b_{k l} \circ c_{t u}\right)$, where $a_{i j}=[A]_{i j}, b_{k l}=$ $[B]_{k l}, c_{t u}=[C]_{t u}$.

Theorem 10. If $(S, \circ)$ is a commutative semi-group, and for $A_{m \times n}, B_{p \times q}, C_{n \times r}$ and $D_{q \times s},(A \otimes B) \circ(C \otimes D)=$ $(A \otimes C) \circ(B \otimes D)$, where $a_{i j}=[A]_{i j}, b_{k l}=[B]_{k l}, c_{t u}=[C]_{t u}, d_{w v}=[D]_{w v}$.

This theorem is equivalent the operate of elements: $\left(a_{i j} \circ b_{k l}\right) \circ\left(c_{t u} \circ d_{w v}\right)=\left(a_{i j} \circ c_{t u}\right) \circ\left(b_{k l} \circ d_{w v}\right)$.

\section{Application of generalized Kronecker product ${ }^{[7],[8],[9],[10],[11]}$}

Theorem 12. Suppose $S=\left\{a_{1}, a_{2}, \cdots, a_{n}\right\}$ and $(S, \circ)$ is an algebraic system, then the table of algebraic operator in $S$ is as follows:

$<$ Figure1 $>$

Construct $A=\left[\begin{array}{llll}a_{1} & a_{2} & \cdots & a_{n}\end{array}\right]^{T}$, then $(S, \circ)$ is a semi-group if and only if $A \otimes\left(A \otimes A^{T}\right)=(A \otimes A) \otimes A^{T}$.

Theorem 13. Suppose $S=\left\{a_{1}, a_{2}, \cdots, a_{n}\right\}$ and $(S, \circ)$ is an algebraic system, then the table of algebraic operator in $S$ is as follows:

$<$ Figure1 $>$

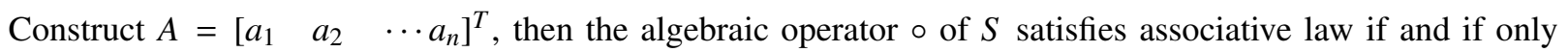
$A \otimes\left(A \otimes A^{T}\right)=(A \otimes A) \otimes A^{T}$.

Sample: Suppose set $S=\{a, b, c, d\}$, the algebraic operator in $S$ is as follows:

$<$ Figure2>

then whether is the algebraic operator $\circ$ in $S$ associative?

Proof: Easily, algebraic operator $\circ$ in finite set $S$ is closed, then $(S, \circ)$ is a algebraic system. Construct matrix $A=\left[\begin{array}{l}a \\ b \\ c \\ d\end{array}\right]$, and the definition of generalized Kronecker product is as definition 3 , then

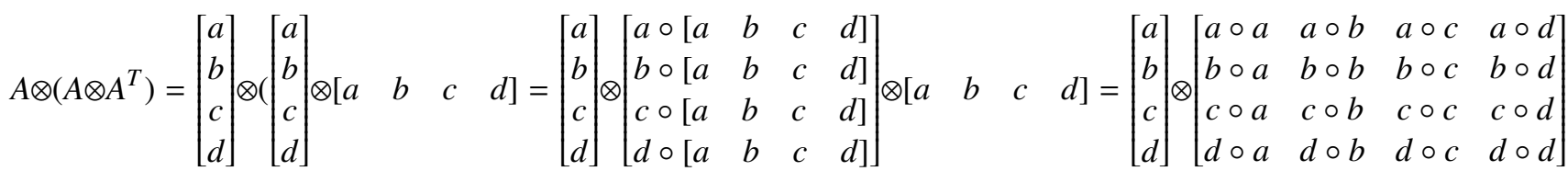

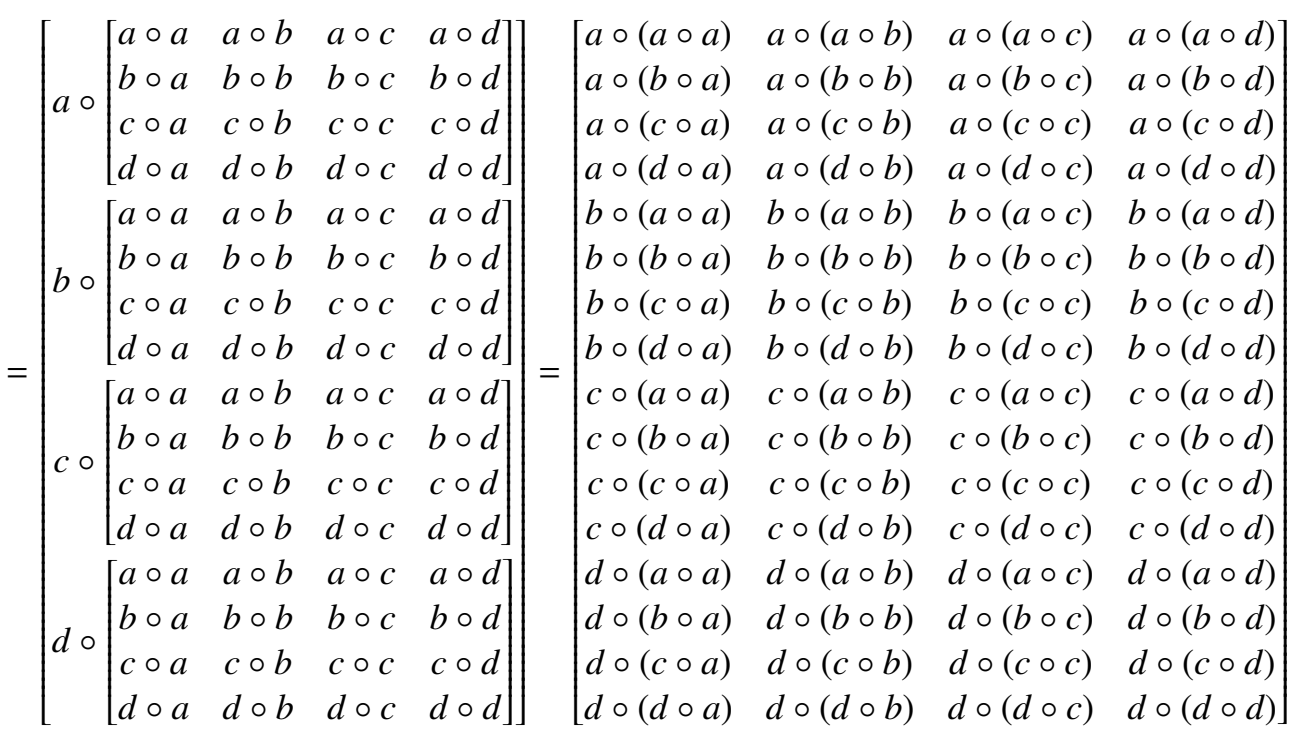




$=\left[\begin{array}{llll}a \circ a & a \circ c & a \circ d & a \circ a \\ a \circ b & a \circ c & a \circ a & a \circ d \\ a \circ c & a \circ a & a \circ b & a \circ d \\ a \circ d & a \circ b & a \circ a & a \circ c \\ b \circ a & b \circ c & b \circ d & b \circ a \\ b \circ b & b \circ c & b \circ a & b \circ d \\ b \circ c & b \circ a & b \circ b & b \circ d \\ b \circ d & b \circ b & b \circ a & b \circ c \\ c \circ a & c \circ c & c \circ d & c \circ a \\ c \circ b & c \circ c & c \circ a & c \circ d \\ c \circ c & c \circ a & c \circ b & c \circ d \\ c \circ d & c \circ b & c \circ a & c \circ c \\ d \circ a & d \circ c & d \circ d & d \circ a \\ d \circ b & d \circ c & d \circ a & d \circ d \\ d \circ c & d \circ a & d \circ b & d \circ d \\ d \circ d & d \circ b & d \circ a & d \circ c\end{array}\right]=\left[\begin{array}{llll}a & d & a & a \\ c & d & a & a \\ a & c & a & d \\ b & a & d & b \\ c & a & b & d \\ a & b & c & d \\ d & c & b & a \\ c & b & d & c \\ a & b & c & d \\ b & c & a & d \\ d & a & c & b \\ d & a & c & d \\ b & a & d & c \\ a & d & b & c \\ c & b & d & a\end{array}\right]$.

But

$$
\left.(A \otimes A) \otimes A^{T}=\left(\begin{array}{l}
a \\
b \\
c \\
d
\end{array}\right] \otimes\left[\begin{array}{l}
a \\
b \\
c \\
d
\end{array}\right]\right) \otimes\left[\begin{array}{llll}
a & b & c & d
\end{array}\right]
$$

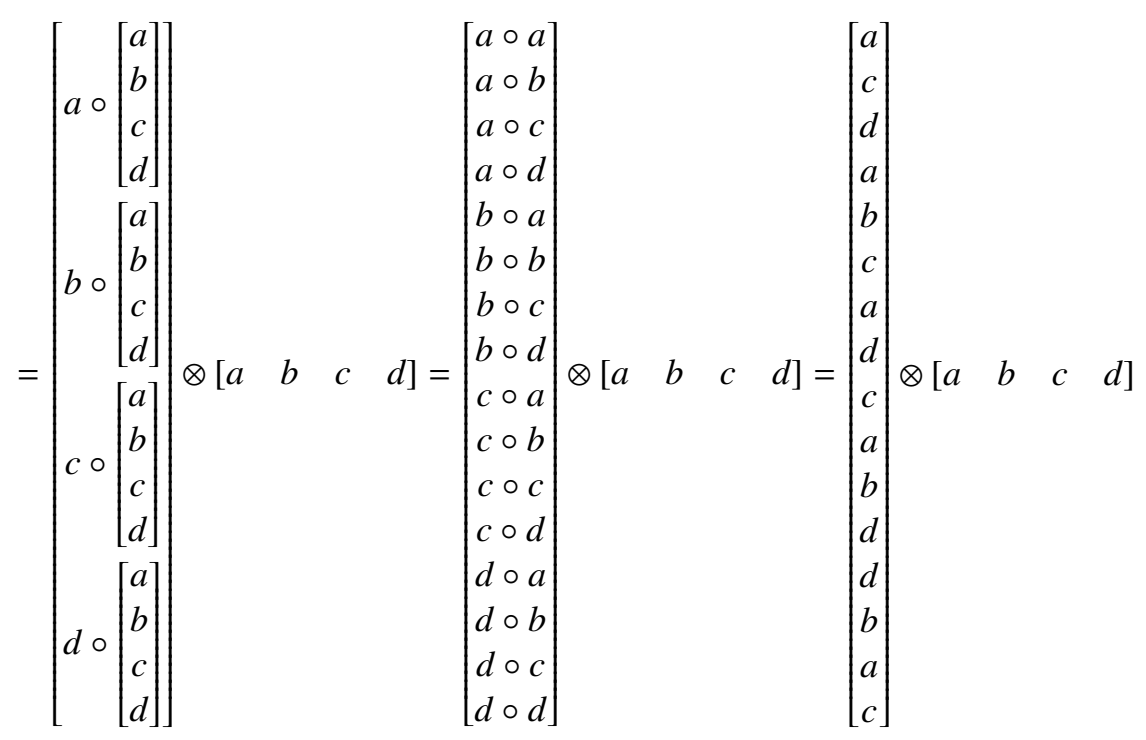




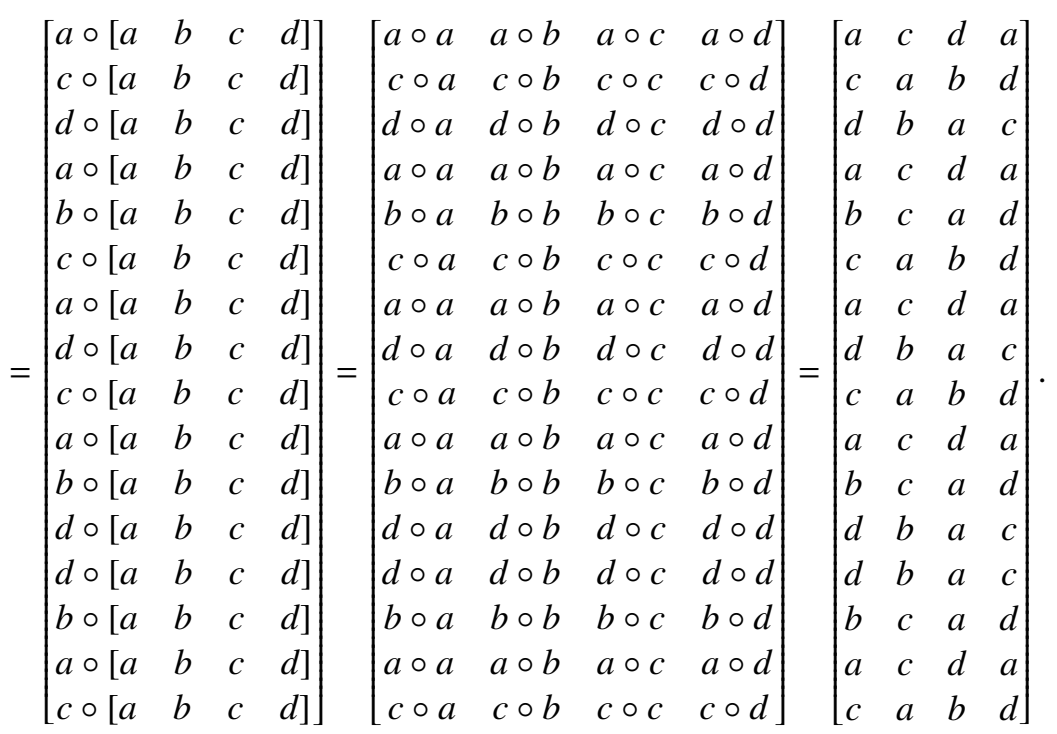

Because

$$
\left[\begin{array}{llll}
a & d & a & a \\
c & d & a & a \\
d & a & c & a \\
a & c & a & d \\
b & a & d & b \\
c & a & b & d \\
a & b & c & d \\
d & c & b & a \\
c & b & d & c \\
a & b & c & d \\
b & c & a & d \\
d & a & c & b \\
d & a & c & d \\
b & a & d & c \\
a & d & b & c \\
c & b & d & a
\end{array}\right] \neq\left[\begin{array}{llll}
a & c & d & a \\
c & a & b & d \\
d & b & a & c \\
a & c & d & a \\
b & c & a & d \\
c & a & b & d \\
a & c & d & a \\
d & b & a & c \\
c & a & b & d \\
a & c & d & a \\
b & c & a & d \\
d & b & a & c \\
d & b & a & c \\
b & c & a & d \\
a & c & d & a \\
c & a & b & d
\end{array}\right] .
$$

$A \otimes\left(A \otimes A^{T}\right) \neq(A \otimes A) \otimes A^{T}$. From theorem 13, the algebraic operator $\circ$ in $S$ is suit for associative law.

\section{References}

Bellman R. (1970). Introduction to Matrix Analysis. New York: McGraw-Hill.

Ltkepohl H. (1996). Handbook of Matrices. New York:John Wiley and Sones.

Rao C R,Mitra S K. (1971). Generalized Inverse of Mateices]. New York: John Wiley and Sones.

Brewer J W. (1978). Kronecker products and matrix calculus in system theory. IEEE Trans Circuits and Systems, 25, 772-781.

Xianda Zhang. (2004), Analysis of matrix and its application. Beijing: tsinghua university press, 107-132.

Andrews H C,Kane J. (1970). Kronecker matrices, computetr implementation, and generalized spectra. J Assoc Comput Mach, 17: 260-268.

Alexander S T. (1986). Adaptive Signal Processing: Theory and Applications. New York: Springer Verlag.

Shaoxue Liu. (1999). Base of Modern Algebra. Beijing: High Education Press.

Pinsan Wu. (1979). Modern Algebra. Beijing: High Education Press.

Guanzhang Hu. (1999). Application Modern Algebra. Beijing: tsinghua university press.

Pingtian Zhu, Bohong Li Etc. (2001) Modern Algebra. Beijing: Scientific Press. 


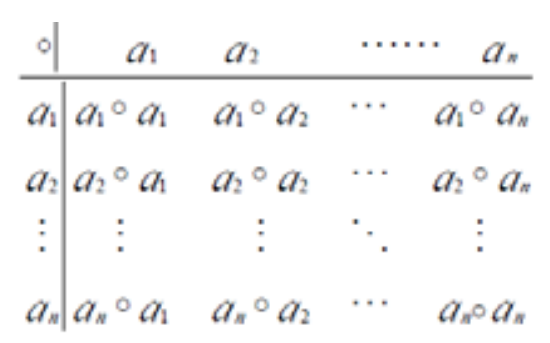

Figure 1

\begin{tabular}{l|llll}
$\circ$ & $a$ & $b$ & $c$ & $d$ \\
\hline$a$ & $a$ & $c$ & $d$ & $a$ \\
$b$ & $b$ & $c$ & $a$ & $d$ \\
$c$ & $c$ & $a$ & $b$ & $d$ \\
$d$ & $d$ & $b$ & $a$ & $c$
\end{tabular}

Figure 2 